\title{
Why Early Prevention of Childhood Obesity Is More Than a Medical Concern: A Health Economic Approach
}

\author{
Diana Sonntag ${ }^{a, b}$ \\ ${ }^{a}$ Mannheim Institute of Public Health (MIPH), Medical Faculty of Heidelberg University, Mannheim, Germany; \\ ${ }^{b}$ Department of Health Sciences, University of York, York, UK
}

\section{Keywords}

Childhood obesity - Economic evaluation - Lifetime cost .

Best-practice toolbox

\begin{abstract}
Background: Childhood overweight and obesity are a nondeniable health concern with increasing economic attention. Summary: International studies provide robust evidence about substantial lifetime excess costs due to childhood obesity, thereby underscoring the urgent need to implement potent obesity prevention programs in early childhood. Fortunately, this is happening more and more, as evidenced by the increase in well-conducted interventions. Nevertheless, an important piece of the puzzle is often missing, that is, health economic evaluations. There are 3 main reasons for this: an insufficient number of economic approaches which consider the complexity of childhood obesity, a lack of (significant) long-term effect sizes of an intervention, and inadequate planning of health economic evaluations in the design phase of an intervention. Key Messages: It is advisable to involve health economists during the design phase of an intervention. Equally necessary is the development of a tailored toolbox for efficient data acquisition.
\end{abstract}

(c) 2017 S. Karger AG, Basel

\section{KARGER}

๑) 2017 S. Karger AG, Basel

E-Mail karger@karger.com

www.karger.com/anm

\section{Introduction}

Childhood overweight and obesity are growing pediatric health concerns worldwide. Although childhood obesity rates are lower in Europe than in the US and Australia, the rates have risen substantially in the last few decades [1]. This increasing prevalence of overweight and obesity in children is particularly alarming due to the immediate development of metabolic imbalances, which lead to increased and severe morbidity in childhood. Moreover, it is also of great concern because overweight and obesity can persist in adulthood [2] creating long-term health and social consequences (i.e., increased risk of obesity-related comorbidities, premature death, and work-related productivity losses) [3]. However, childhood overweight and obesity are more than a medical concern; they are increasingly an economic one. This is mainly due to their substantial cost burden, particularly later in life. Childhood overweight and obesity result in direct and indirect costs. The direct costs are predominantly healthcare ones due to an increased risk of developing obesity-associated diseases that need treatment, while the indirect costs are related to sick leave, reduced productivity, and premature death. 
Table 1. Estimated direct and indirect lifetime costs of overweight and obesity, per sex $[5,7]$

\begin{tabular}{|c|c|c|c|c|}
\hline & \multicolumn{2}{|l|}{ direct } & \multicolumn{2}{|l|}{ indirect } \\
\hline \multicolumn{5}{|l|}{ Male } \\
\hline Difference normal weight vs. obese (excess cost) & 14,524 & 4,262 & 11,557 & 4,209 \\
\hline Cost increase factor (if obese/if normal weight as child) & 3 & 2 & 4 & 3 \\
\hline Proportion occuring beyond age 60 years & - & 0.44 & - & 0.19 \\
\hline \multicolumn{5}{|l|}{ Female } \\
\hline If obese as child & 27,673 & 8,654 & 8,692 & 3,140 \\
\hline
\end{tabular}

\section{The Economic Burden of Childhood Overweight and Obesity}

A number of cost of illness (COI) studies [4] have shown that overweight and obesity during adulthood cause substantial direct and indirect costs, while neglecting to take into account the increased risk of mortality and obesity-related co-morbidities due to childhood obesity. As a result, long-term healthcare consequences and costs cannot be estimated. Only a few international COI studies [5-7] have estimated the lifetime cost of childhood overweight and obesity. Fernandes [6], for example, calculated lifetime costs among the US elementary school population aged 6-11 in 2008, estimating them to be $\$ 31,869$ for obese boys and $\$ 39,815$ for obese girls. When compared with normal weight adults, lifetime excess costs were more than twice or thrice as high among men or women, respectively, in adults who had been obese during childhood [6]. Only 2 pediatric simulation studies in Germany $[5,7]$ considered the long-run economic impact of childhood overweight and obesity, estimating total lifetime costs of $€ 1.8$ billion for the current prevalent population (discounted at $3 \%$, cost year 2010). More specifically, Table 1 shows that costs attributable to overweight and obesity are 3 times higher for men and nearly 5 times higher for women with a history of childhood obesity. Compared to adults who were normal weight as children, expected lifetime excess costs for adults who were overweight or obese during childhood were $€ 8,471$ for men and $€ 9,473$ for women. A striking pattern is that most lifetime excess costs for wom- en occur in the healthcare sector $(€ 6,333)$ while indirect costs attributable to overweight and obesity are much smaller $(€ 2,445)$. The relatively lower estimate of expected indirect lifetime costs is likely due to a lower rate of fulltime employment in women (mostly because of household responsibilities) and lower wage rates. Table 1 also shows that childhood obesity is the top contributor to the overall cost burden across all decades of life. More specifically, compared to adults who were normal weight as children, costs are 8 times higher for adults (between 41 and 51 years) with a history of childhood obesity.

Given the high lifetime excess costs of childhood overweight and obesity, an obvious policy question is how changing the current childhood obesity trend would affect lifetime costs. For example, Sonntag et al. [5] estimated that if the prevalence of childhood obesity were reduced by $1 \%$ in Germany, the expected reduction in lifetime excess cost would be $€ 4.1$ million for the current prevalent population. Expected lifetime costs could be further reduced by $€ 27$ million if the prevalence of childhood overweight and obesity could be reduced by $14 \%$ (which is potentially achievable based on a national evaluation study of outpatient and inpatient care in Germany, EvAKuJ study [8]). Moreover, Sonntag et al. [5] demonstrated that larger effect sizes of obesity interventions in early childhood would result in much higher cost savings. For instance, in an optimistic scenario, Sonntag et al. [5] assumed a drastic reduction of the prevalence of overweight and obesity at the level of the year 1999 and estimated cost savings of approximately $€ 68$ million in Germany. 


\section{Economic Evaluation of Obesity Prevention in Childhood - Results, Limitations, and Best Practice Guidelines}

While there is a growing body of literature about the effectiveness of interventions in the field of childhood obesity prevention [9], relatively few studies have assessed whether the benefits of an intervention justify costs. Most of these studies evaluate the economic consequences of school programs, finding that they were indeed cost-effective. However, the number of cost-effectiveness studies targeting preschool children is small [10], with only a few economic evaluations of infant feeding as early obesity prevention. For the US, for example, Bartick and Reinhold [11] estimated that compared to formula, breastfeeding would result in substantial cost savings. Yet, this approach is shortsighted since their economic evaluation does not take into account effects such as the increased risk of becoming overweight or obese due to the higher protein content in formula [12].

There are numerous reasons why only a small number of economic evaluations of obesity prevention in childhood have been published. First, given the complexity of childhood obesity and key obesogenic environments, conventional approaches commonly used in economic evaluations during adulthood may be of limited use [10]. For instance, Sonntag et al. [13] have shown that obesityrelated dietary behavior in childhood is influenced not only by parents but also by 5 key obesogenic environments: schools, television, the internet, retailers, and advertising campaigns. A comprehensive economic evaluation must not merely consider the economic consequences of parental behavior on children; it must also capture whether obesity prevention programs mitigate negative effects of key obesogenic environments and how this can be monetarily evaluated. It is therefore essential to develop a new framework for economic evaluations that goes beyond evaluating randomized control trials. Second, only few interventions have proven to be (significantly) effective in preventing overweight and obesity, particularly when looking at long-term effectiveness [10]. While small effect sizes are clinically less important, economic evaluations are still of great relevance. For instance, even if one intervention is ineffective, it may have a significantly lower cost compared to another intervention and can be thus considered a cost-reducing strategy. Finally, health economic evaluations are often not planned from the beginning of an intervention, making it difficult for intervention developers, implementers, and health economists to coordinate their efforts effectively. Thus, retrospective evaluations tend to be imprecise since they rely on assumed data and not on the data measured during the intervention. Moreover, since the effects of interventions early in childhood are, if ever, only visible decades after the intervention has taken place, a comprehensive cost-effectiveness analysis requires data collection beyond the end of a trial. It is therefore advisable to involve health economists during the phases of intervention design and data collection. More importantly, developing a toolbox that includes best practice guidelines for clinicians, implementers, and healthcare specialists would be of great relevance to collect critical health economic data. While effects of interventions are generally captured in a standardized way using measures as weight, body mass index (BMI), or BMI z-scores, costs are often measured in a non-standardized way. It is thus difficult to compare these costs because of the heterogeneity of cost categories included in questionnaires.

\section{Conclusions}

With childhood overweight and obesity, a non-deniable health and economic concern; it is vital that obesity prevention be offered as early as possible. Fortunately, this is happening more and more, as evidenced by the increase in well-conducted and evaluated interventions in the field of early childhood obesity prevention. Nevertheless, an important piece of the puzzle is often missing, that is, health economic evaluations. Thus, it is advisable to involve health economists during the design phase of an obesity prevention intervention and to develop a tailored toolbox for efficient data acquisition.

\section{Disclosure Statement}

The author has no conflict of interest to disclose. The author has no financial relationships relevant to this article to disclose.

\begin{tabular}{|c|c|}
\hline References & $\begin{array}{l}1 \text { Wang Y, Lobstein T: Worldwide trends in } \\
\text { childhood overweight and obesity. Int J Pedi- } \\
\text { atr Obes 2006;1:11-25. } \\
2 \text { Singh AS, Mulder C, Twisk JW, van Mechelen } \\
\text { W, Chinapaw MJ: Tracking of childhood over- } \\
\text { weight into adulthood: a systematic review of } \\
\text { the literature. Obes Rev 2008;9:474-488. } \\
3 \text { Caird J, Kavanagh J, O’Mara-Eves A, Oliver } \\
\text { K, Oliver S, Stansfield C, Thomas J: Does be- } \\
\text { ing overweight impede academic attainment? } \\
\text { A systematic review. Health Educ J 2014;73: } \\
\text { 497-521. }\end{array}$ \\
\hline
\end{tabular}


4 Tsai AG, Williamson DF, Glick HA: Direct medical cost of overweight and obesity in the USA: a quantitative systematic review. Obes Rev 2011;12:50-61.

5 Sonntag D, Ali S, De Bock F: Lifetime indirect cost of childhood overweight and obesity: a decision analytic model. Obesity (Silver Spring) 2016;24:200-206.

6 Fernandes MM: Estimating the lifecycle costs associated with childhood obesity in: evaluating the impacts of school nutrition and physical activity policies on child health (dissertation). RAND COOPERATION, 2010.

7 Sonntag D, Ali S, Lehnert T, Konnopka A, Riedel-Heller S, König HH: Estimating the lifetime cost of childhood obesity in Germany: results of a Markov Model. Pediatr Obes 2015; $10: 416-422$.
8 Hoffmeister U, Bullinger M, van EgmondFroehlich A, Goldapp C, Mann R, RavensSieberer U, et al: [Overweight and obesity in childhood and adolescence. Evaluation of inpatient and outpatient care in Germany: the EvAKuJ study]. Bundesgesundheitsblatt Gesundheitsforschung Gesundheitsschutz 2011;54:128-135.

9 Blake-Lamb TL, Locks LM, Perkins ME, Woo Baidal JA, Cheng ER, Taveras EM: Interventions for childhood obesity in the first 1,000 days a systematic review. Am J Prev Med 2016;50:780-789.

10 Döring N, Mayer S, Rasmussen F, Sonntag D: Economic evaluation of obesity prevention in early childhood: methods, limitations and recommendations. Int J Environ Res Public Health 2016;13:pii:E911.
11 Bartick M, Reinhold A: The burden of suboptimal breastfeeding in the United States: a pediatric cost analysis. Pediatrics 2010;125: e1048-e1056.

12 Weber M, Grote V, Closa-Monasterolo R, Escribano J, Langhendries JP, Dain E, Giovannini M, Verduci E, Gruszfeld D, Socha $\mathrm{P}$, et al: Lower protein content in infant formula reduces BMI and obesity risk at school age: follow-up of a randomized trial. Am J Clin Nutr 2014;99:10411051.

13 Sonntag D, Schneider S, Mdege N, Ali S, Schmidt B: Beyond food promotion: a systematic review on the influence of the food industry on obesity-related dietary behaviour among children. Nutrients 2015;7:85658576. 